\title{
Caracterização fisíco-química e análise microbiológica de cookie de farinha de aveia
}

\author{
Bruna Ferreira Dias¹, Gabriela Silva Santana², Ellen Godinho Pinto², Camila Fernanda Dias \\ de Oliveira ${ }^{1}$
}

\author{
${ }^{1}$ Universidade Federal de Goiás, Campus Samambaia, Goiânia, Goiás, Brasil. E-mail: brunaferreira142011@hotmail.com, \\ camilaferdias@gmail.com \\ ${ }^{2}$ Instituto Federal Goiano, Campus Morrinhos, Morrinhos, Goiás, Brasil. Email:gabysantana_92@hotmail.com, \\ ellen.godinho@ifgoiano.edu.br
}

Recebido: 15/04/2016; Aceito: 07/07/2016.

\section{RESUMO}

A aveia é uma gramínea, pertencente à família Poaceae e ao gênero Avena sp. Seu grão é amplamente utilizado para a fabricação de produtos de panificação. O biscoito é um produto que apresenta grande interesse comercial e está em constante desenvolvimento. Com base nos estudos, o objetivo no trabalho foi explorar o efeito da incorporação da farinha de aveia no processamento de biscoito tipo cookie, com relação às características físicoquímicas e microbiológicas. Foi elaborado biscoito tipo cookie enriquecido com farinha de aveia, com diferentes concentrações de aveia $(2 \%, 4 \%, 6 \%)$. Estes foram avaliados quanto às características físico-químicas (pH, sólidos solúveis totais, vitamina $\mathrm{C}$ e umidade) e microbiológica (coliformes totais). A partir das análises físico-químicas realizadas nos biscoitos foi possível verificar que houve diferença significativa apenas nas amostras de vitamina $\mathrm{C}$, umidade e no teor de sólidos solúveis totais ao nível de 5\% de significância, entre os biscoitos tipo cookie de aveia.

Palavras-chave: Aveia, vitamina C, cookie.

\section{Physico-chemical and microbiological characterization of oat flour cookie}

\begin{abstract}
Oats is a grass belonging to Poaceae family and genus Avena sp. Its grain is widely used for manufacturing bakery products. The cookie is a product that has great commercial interest and is constantly developing. Based on studies, the work aimed to explore the effect of oat flour incorporation into the biscuit processing type cookie, with respect to characteristics of physical, chemical and microbiological. Biscuit was prepared cookie type enriched with oat flour, oat with different concentrations $(2 \%, 4 \%, 6 \%)$. These were evaluated for physicochemical characteristics (pH, soluble solids, vitamin $\mathrm{C}$ and humidity) and microbiological (total coliform). From the physical and chemical analyzes in the cookies we found that there was a significant difference only in samples of vitamin $\mathrm{C}$, humidity and total soluble solids content at 5\% significance level, between the cookies type cookie oat flour and in microbiological analysis showed absence of coliforms.
\end{abstract}

Key words: Oat, vitamin C, cookie. 


\section{Introdução}

Os recentes estudos vêm evidenciando a relação entre dieta e saúde, somados ao crescente interesse de alguns indivíduos em consumir alimentos mais "saudáveis", têm levado a indústria alimentícia ao desenvolvimento de novos produtos cujas funções almejam ir além do fornecimento de nutrientes básicos e da satisfação do paladar do consumidor. Esses produtos são conhecidos como "alimentos funcionais" e têm como principal função a prevenção do risco de doenças crônico-degenerativas (SCARAMAL; ALMEIDA, 2008; DURANTE, 2011).

Tendo em aspecto a demanda da indústria alimentícia pelo desenvolvimento de novos produtos, com destaque no mercado consolidado de biscoitos e no interesse dos consumidores por produtos com valor nutricional e/ou benefícios para a saúde são de grande proeminência a elaboração do cookie de farinha de aveia (SOUZA, 2001).

Biscoito é o produto adquirido pelo amassamento e cozimento da massa preparada com farinhas, amidos, fermentada ou não e outras substâncias alimentícias. Sua qualidade está relacionada com o sabor, a textura, a aparência entre outros fatores, e nos últimos anos vem se destacando como um produto de grande interesse comercial em decorrência de sua praticidade na produção, comercialização e consumo, além de possuir longa vida comercial (SANTOS, 2011).

Os biscoitos tipo cookies têm boa aceitação sensorial e comercial, sendo muito contemplados por crianças e adultos, e ainda possuem vida de prateleira relativamente longa. Muitos autores elaboraram cookies com redução de gordura, adição de farinha de aveia (GUTKOSKI et al., 2007), farinha de soja e farelo de aveia (MARETI et al., 2010), farelo de trigo e arroz (FEDDERN et al., 2011), grãos de leguminosas (ZUCCO et al., 2011), farinha integral de cevada (GUPTA et al., 2011) e farinha de banana verde (AGAMA-ACEVEDO et al., 2012).

A aveia é uma gramínea, pertencente à família Poaceae e ao gênero Avena. Seu grão é amplamente utilizado para a fabricação de produtos de panificação, com o objetivo de melhorar os teores de fibra alimentar, pois contém uma quantidade considerada de fibras em relação aos demais cereais, alcançando assim, uma boa aceitação pelo consumidor, relacionado à diminuição dos níveis de colesterol e riscos de doenças coronárias. Por isso, tem crescido o interesse dos consumidores por produtos que contenham este grão em sua formulação (SANTOS, 2011).

Os biscoitos tipo cookie possuem vários atrativos como: grande consumo, vida útil relativamente longa e boa aceitação, particularmente entre crianças. Recentemente, os biscoitos tipo cookie tem sido formulados com a intenção de implementar sua fortificação com fibra ou proteína, devido ao forte apelo nutricional que existe hoje em dia com relação aos alimentos consumidos (GARMUS, 2009).

A utilização de farinhas mistas expandiu-se, sendo utilizada na fabricação de biscoitos, já que este é um produto altamente aceito e consumido por pessoas de todas as faixas etárias. Tais características, aliadas à sua enorme diversidade, apresentam-se como uma nova opção para o estudo de diferentes tipos de farinhas e suas propriedades físicas, químicas e sensoriais, possibilitando o aumento das propriedades tecnológicas e funcionais (SILVA et al., 2001; KOPPER et al., 2009).

O objetivo no trabalho foi avaliar o efeito da incorporação da farinha de aveia no processamento de biscoito tipo cookie, com relação às características físico-químicas e microbiológicas do produto.

\section{Material e Métodos}

Foram utilizadas como matérias-primas adquiridos em comércio local do município de Morrinhos- GO. Logo em seguida foram elaborados os biscoitos cookie no próprio laboratório da Agroindústria e todas as análises físicas, químicas e microbiológicas foram realizadas no laboratório de Microbiologia do Instituto Federal Goiano, Campus- Morrinhos.

A elaboração dos biscoitos cookie foi adaptada da metodologia proposta por Piovesana et al. (2013). Foram realizados em proporções diferentes, 2\%, 4\% e $6 \%$ de farinha de aveia seguindo a Tabela 1. Os ingredientes foram misturados em batedeira elétrica. As massas dos biscoitos cookie foram submetidas ao forneamento a $120^{\circ} \mathrm{C}$ por 15 minutos.

Todas as análises físico-químicas foram realizadas em triplicata e os resultados expressos como média e desvio padrão. A quantificação dos teores vitamina $\mathrm{C}$, foi determinada segundo a metodologia do Instituto Adolfo Lutz (2004) com iodato de potássio.

A umidade foi realizada pelo método gravimétrico de volatilização, por secagem direta em estufa a $105^{\circ} \mathrm{C}$ até a obtenção do peso constante. $\mathrm{O}$ pH foi determinado em potenciômetro calibrado com solução padrão 4 e 7 para melhores resultados. Sólidos solúveis totais realizados por medida direta por refratômetro todos os métodos descritos pelo Instituto Adolfo Lutz (2004).

Análise microbiológica foi realizada de acordo com o Regulamento Técnico sobre Padrões Microbiológicos para Alimentos (BRASIL, 2001). Seguindo a técnica de números mais provável (NMP) de acordo com esse regulamento deverá conter pesquisa de coliformes a 45 ${ }^{\circ} \mathrm{C} \mathrm{g}^{-1}$ todas realizadas em triplicata. 
Tabela 1. Ingredientes e quantidades em porcentagem utilizadas na elaboração de amostra com FA2, FA4 e FA6 de aveia dos biscoitos tipo cookie de aveia.

\begin{tabular}{lccc}
\hline Ingredientes & Quantidade $\%$ & Quantidade $\%$ & \multicolumn{2}{c}{ Quantidade $\%$} \\
\hline Farinha de trigo & FA2 & FA4 & 31 \\
Farinha de aveia & 35 & 33 & 13,8 \\
Margarina & 4,57 & 9,17 & 13 \\
Açúcar refinado & 13 & 13 & 30 \\
Sal & 35 & 33 & 0,44 \\
Fermento químico & 0,46 & 0,44 & 0,91 \\
Ovo & 0,92 & 0,91 & 11,40 \\
\hline
\end{tabular}

FA2: Formulação com 2\% de farinha de aveia; FA4: Formulação com 4\% de farinha de aveia; FA6: Formulação com 6\% de farinha de aveia.

Os resultados da caracterização física e química foram submetidos à Análise de Variância (ANOVA) e teste de média de Tukey, a $5 \%$ de probabilidade utilizando o programa de estatística ASSISTAT versão 7.0 .

\section{Resultados e Discussão}

Os resultados das amostras de biscoito tipo cookie nas concentrações de $4 \%$ e $6 \%$ diferiram entre si para o teor de vitamina $\mathrm{C}$ e sólidos solúveis, entretanto na concentração $2 \%$ diferiu na umidade e teor de sólidos solúvel entre as demais, porém todas apresentaram dentro das especificações proposto pela legislação brasileira (BRASIL, 2001).

Os biscoitos cookie elaborados com a farinha de aveia apresentaram umidade entre 2,0 a $4,9 \%$, valores situados dentro dos teores de umidade especificados pela legislação brasileira, que deve ser inferior a $14 \%$. Finco et al. (2009) elaboraram um biscoito semelhante com a adição de farinha de berinjela e a umidade dos biscoitos foi superior a este estudo com $12,5 \%$.

Menores percentuais de umidade são ideais para um aumento da sua vida de prateleira (Madrona; Almeida, 2008), já que baixos conteúdos de umidade são capazes de inibir o crescimento de microrganismos e provocar modificações na textura.

Em relação ao $\mathrm{pH}$, as formulações não se diferiram entre si estatisticamente apresentando valores entre 7,6 a 7,8 e enquadrados na faixa normal para biscoitos (entre 6,5 e 8,0), conforme Maciel et al. (2008), observaram nos testes realizados em biscoitos tipo cookie.
A análise de sólidos solúveis totais do biscoito com farinha de aveia indicou um teor médio entre $27^{\circ}$ a 33 ${ }^{\circ}$ Brix, enquanto Aquino et al. (2010), encontraram um valor para sólidos solúveis totais igual a $47,5^{\circ}$ Brix para biscoitos tipo cookies elaborados com farinha de resíduos de acerola.

No Brasil, a ingestão diária, recomendada (IDR) pela Resolução RDC $n^{\circ} 269$, de setembro de 2005 , de vitamina $C$ para crianças de 4 a 10 anos é de $45 \mathrm{mg}$ e para adultos é de $60 \mathrm{mg}$, e esta quantidade pode ser fornecida pela ingestão de, aproximadamente, 8 cookies enriquecidos com farinha de aveia nas concentrações de $2 \%$ e $4 \%$ para uma alimentação de adultos.

Devido a essa característica, o cookie enriquecido com aveia pode se tornar interessante para a ingestão necessária de vitamina $\mathrm{C}$, sabendo que o ácido ascórbico desempenha várias funções biológicas relacionadas ao sistema imune, formação de colágeno, absorção de ferro, inibição da formação de nitrosaminas e atividade antioxidante. Pode-se observar nas análises de vitamina $C$, para as concentrações $2 \%$ e $4 \%$ não si diferiram entre si, somente na concentração $6 \%$ apresentou diferença significativa.

$\mathrm{Na}$ Tabela 2 estão os resultados das análises físicoquímicas, para cookie de aveia com concentrações $2 \%$, $4 \%$, e $6 \%$. Os resultados microbiológicos apresentados na Tabela 3 para coliformes a $45{ }^{\circ} \mathrm{C}$ foram ausentes. De acordo com os resultados podemos considerar que os biscoitos tipo cookie estão aptos ao consumo, pois estão dentro dos padrões estabelecidos pela legislação vigente demonstrando assim, que foram manipulados de forma adequada (BRASIL, 2001).

Tabela 2. Análises Físico-químicas de cookie de aveia em diferentes concentrações de aveia

\begin{tabular}{lccc}
\hline & FA2 & FA4 & FA6 \\
\hline $\mathrm{pH}$ & $7,6 \pm 0,05^{\mathrm{a}}$ & $7,8 \pm 0,05^{\mathrm{a}}$ & $7,8 \pm 0,05^{\mathrm{a}}$ \\
Umidade $(\%)$ & $2,0 \pm 0,55^{\mathrm{b}}$ & $4,2 \pm 0,4^{\mathrm{a}}$ & $4,9 \pm 0,3^{\mathrm{a}}$ \\
Vitamina C (mg/100g) & $10,5 \pm 0^{\mathrm{a}}$ & $10,5 \pm 0^{\mathrm{a}}$ & $30 \pm 0^{\mathrm{b}}$ \\
Sólidos Solúvel Totais & $33 \pm 0^{\mathrm{a}}$ & & $27 \pm 0^{\mathrm{c}}$ \\
$\left({ }^{\circ}\right.$ Brix) & & & \\
\hline
\end{tabular}

*Médias seguidas pela mesma letra em cada linha não diferem entre si a $\mathrm{p} \leq 0,05$. 
Zuniga et al. (2011), obtiveram resultados semelhantes ao analisar amostras de biscoitos de castanha de caju tipo integral, demonstrando que nenhuma unidade apresentou contagem positiva para coliformes. O mesmo resultado também foi obtido por Krüger et al. (2003), para formulação de biscoitos tipo "cookie" e "snack" enriquecido.

\section{Conclusões}

Por meio dos resultados obtidos pode-se concluir que o objetivo do presente trabalho foi atingido, tendo sido elaborados biscoitos tipo cookie enriquecidos com farinha de aveia. Pode-se observar que é possível obter biscoitos com características desejáveis através da incorporação da farinha de aveia na fabricação de biscoito tipo cookie. Essas características desejadas podem ser observadas através dos resultados obtidos nas análises fisíco-quimicas e análises microbiológicas. Os biscoitos apresentaram-se aptos para o consumo não apresentando riscos a saúde do consumidor, atestando a eficiência e higiene na elaboração do produto.

\section{Referências Bibliográficas}

AGAMA-ACEVEDO, E.; ISLAS-HERNANDEZ, J. J. PACHECO-VARGAS, et al. Starch digistibility and glycemic index of cookies parcially substituted with unripe banana flour. Food Science and Technology, Amsterdam, v. 46, n. 2, p.177-182, 2012.

AQUINO, A. M. S.; Avaliação físico-química e aceitação sensorial de biscoitos tipo cookies elaborados com farinha de resíduos de acerola. Revista Instituto Adolfo Lutz, São Paulo-SP, v.18, n. 03 p.150, 2010.

BRASIL. Resolução RDC $\mathbf{n}^{\circ}$ 269, de setembro de 2005 . Aprova o Regulamento Técnico sobre a ingestão diária recomendada (IDR) de proteína, vitaminas e minerais. Disponível: <http://www.anvisa.org.br> Acesso em 20.dez. 2015.

BRASIL. Resolução RDC $\mathbf{n}^{0}$ 12, de 02 de janeiro de 2001. Estabelece regulamento técnico sobre os padrões microbiológicos para alimentos. Disponível: <http://www.anvisa.org.br> Acesso em 20.dez. 2013.

DURANTE, V. V. O.; MIRANDA, M. Z.; Avaliação física e sensorial de biscoitos tipo cookie adicionados de farelo de trigo e arroz. Brazilian Journal Food Technology, Campinas-SP, v. 14, n. 4, p. 265-272, 2011

FEDDERN, V.; DURANTE, V. V. O.; MIRANDA, M. Z. Avaliação física e sensorial de biscoitos tipo cookie adicionados de farelo de trigo e arroz. Brazilian Journal of Food Technology, Campinas-SP, v. 14, n. 4, p. 267-274, 2011.

FINCO, A. M. O., BEZERRA, J. R. M. V.; RIGO, M., CÓRDOVA, R. V. Elaboração de biscoitos com adição de farinha de berinjela. Revista Brasileira de Tecnologia Agroindustrial, São Paulo-SP, v. 03, n. 01, p. 49-59, 2009.

GARMUS, T. T.; BEZERRA, J. R. M. V.; RIGO, M.; CÓRDOVA, K. R. V. Elaboração de biscoitos com adição de farinha de casca de batata (Solanum tuberosum L.). Revista Brasileira de Tecnologia Agroindustrial, Paraná, v. 03, n. 02, p. 56-65, 2009.

GUPTA, M.; BAWA, A. S.; ABU-GHANNAM, N. Effect of barley flour and freeze-thaw cycles on textural nutritional and functional properties of cookies. Food and Bioproducts Processing, Amsterdam, v. 89, n. 01, p. 520-527, 2011.

GUTKOSKI, L. C.; IANISKI, F.; DAMO, T. V.; PEDÓ, I. Biscoitos de aveia tipo "cookie" enriquecidos com concentrado de $\beta$-glicanas. Brazilian Journal of Food Technology, Campinas-SP, v. 10, n. 2, p. 104-110, 2007.

KOPPER, A. C.; SARAVIA, A. P. K.; RIBANI, R. H.; LORENZI, G. M. A. C. Utilização tecnológica da farinha de bocaiúva na elaboração de biscoitos tipo cookie. Alimentos e Nutrição, Araraquara-SP, v. 20, n. 3, p. 463-469, 2009.

KRÜGER, C. C. H.; COMASSETTO, M. C. G.; CÂNDIDO, L. M. B.; BALDINI, V. L. S.; SANTTUCCI, M. C.; SGARBIERI, V.C. Biscoitos tipo "cookie" e "snack" enriquecidos, respectivamente com caseína obtida por coagulação enzimática e caseinato de sódio. Ciência e Tecnologia de Alimentos, Campinas-SP, v. 1, n. 23, p. 81-86, 2003.

MACIEL, L. M. B.; PONTES, D. F.; RODRIGUES, M. C. P. Efeito da adição de farinha de linhaça no processamento de biscoito tipo cracker. Revista Alimentos e Nutrição, Araraquara-SP, v. 19, n. 4, p. 385-392, 2008.

MADRONA, G. S.; ALMEIDA, A. M. Elaboração de biscoitos tipo cookie à base de okara e aveia. Revista Tecnológica, São Paulo-SP, v. 17, n. 2, p. 61-72, 2008.

MARETI, M. C.; GROSSMANN, M. V. E.; BENASSI, M. T. Características físicas e sensoriais de biscoitos com farinha de soja e farelo de aveia. Ciência e Tecnologia de Alimentos, Campinas-SP, v. 30, n. 4, p. 878-883, 2010

INSTITUTO ADOLFO LUTZ. Normas Analíticas do Instituto Adolfo Lutz Métodos químicos e físicos para análise de alimentos. $4^{\mathrm{a}}$ ed, São Paulo, IV - IAL, 2004.

PIOVESANA, A. BUENO, M. M..; KLAJN, V. M. Elaboração e aceitabilidade de biscoitos enriquecidos com aveia e farinha de bagaço de uva. Brazilian Journal Food Technology, Campinas-SP, v. 16, n. 1, p. 68-72, 2013.

SANTOS, C. A.; RIBEIRO, R. C.; SILVA, E. V. C.; SILVA, N.; SILVA, B. A. Elaboração de biscoito de farinha de buriti (Mauritia flexuosa L. f) com e sem adição de aveia (Avena sativa L.). Revista Brasileira de Tecnologia Agroindustrial, Ponta Grossa-PR, v. 5, n. 1, p. 262-275, 2011.

SCARAMAL, G. M.; ALMEIDA, A. M. Elaboração de biscoitos tipo cookie à base de okara e aveia. Revista Tecnológica, Campinas-SP, v. 17, n. 2, p. 61-72, 2008.

ILVA, M. R.; SILVA, M. S.; MARTINS, K. A.; BORGES, S. Utilização tecnológica dos frutos de jatobá-do-cerrado e de 
jatobá-da-mata na elaboração de biscoitos fontes de fibra alimentar e isentos de açúcares. Ciência e Tecnologia de Alimentos, Campinas-SP, v. 21, n. 2, p. 176-182, 2001.

SOUZA, M. L.; RODRIGUES, R. S.; FURQUIM, M. F. G.; EL-DASH, A. A. Processamento de "cookies" de Castanhado-Brasil. Boletim do Centro de Pesquisa de Processamento de Alimentos, Curitiba-PR, v. 19, n. 2, p.381390, 2001.

ZUCCO, F.; BORSUK, Y.; ARNTFIELD, S. D. Physical and nutritional evaluation of wheat cookies supplemented with pulse flours of different particle sizes. LWT - Food Science and Technology, Amsterdam, v. 44, n. 1, p. 2070-2076, 2011.

ZUNIGA, A. D. G.; COELHO, A. F. S.; FERREIRA, E. S.; RESENDE, E. A.; ALMEIDA, K. N. Avaliação da vida de prateleira de biscoito de castanha de caju tipo integral. Revista Brasileira de Produtos Agroindustriais, Campina Grande, v.13, n.3, p.251-256, 2011. 